ARTICLE

https://doi.org/10.1038/s41467-020-18673-5

\title{
Optoribogenetic control of regulatory RNA molecules
}

Sebastian Pilsl', Charles Morgan', Moujab Choukeife ${ }^{1}$, Andreas Möglich (1D ${ }^{2}$ \& Günter Mayer (1) 1,3凶

Short regulatory RNA molecules underpin gene expression and govern cellular state and physiology. To establish an alternative layer of control over these processes, we generated chimeric regulatory RNAs that interact reversibly and light-dependently with the lightoxygen-voltage photoreceptor PAL. By harnessing this interaction, the function of micro RNAs (miRs) and short hairpin (sh) RNAs in mammalian cells can be regulated in a spatiotemporally precise manner. The underlying strategy is generic and can be adapted to neararbitrary target sequences. Owing to full genetic encodability, it establishes optoribogenetic control of cell state and physiology. The method stands to facilitate the non-invasive, reversible and spatiotemporally resolved study of regulatory RNAs and protein function in cellular and organismal environments.

\footnotetext{
${ }^{1}$ Life and Medical Sciences (LIMES), University of Bonn, Gerhard-Domagk-Str.1, 53121 Bonn, Germany. ${ }^{2}$ Lehrstuhl für Biochemie, Photobiochemie, University of Bayreuth, Universitätsstraße 30, 95440 Bayreuth, Germany. ${ }^{3}$ Center of Aptamer Research \& Development, University of Bonn, Gerhard-Domagk-Str. 1, 53121 Bonn, Germany. ${ }^{凶}$ email: gmayer@uni-bonn.de
} 
S hort regulatory RNA molecules such as endogenous micro RNAs (miR) or synthetic short hairpin RNAs (shRNA) are essential mediators of gene expression ${ }^{1-3}$. They interact with defined complementary sites in the untranslated (UTR) or the coding regions of mRNA molecules, upon which translation is either inhibited or the mRNA is hydrolyzed. Regulatory RNAs have become indispensable in the biosciences for the validation of gene or protein function in cells and in vivo ${ }^{4}$. Although the ondemand control of mRNA translation has been achieved at the levels of mRNA stability and ribosome processing, e.g., by introducing aptazymes or aptamers in the UTRs ${ }^{5-9}$, the direct control of the function of short regulatory RNAs, ultimately in a spatiotemporal manner, remains challenging. At the same time, it is highly demanded, as it would offer programmable, modular and generalizable control of target gene expression on the posttranscriptional level ${ }^{10-12}$. To this end, small-molecule-responsive siRNAs whose function can be controlled by theophylline or tetracycline $^{13}$ or conditional expressions systems of shRNAs ${ }^{14}$ have been reported. These approaches extend towards the transcriptional regulation of miRs ${ }^{15}$ or to aptazymes that control miR maturation in response to small molecules ${ }^{16}$. Atanasov et al. constructed pre-miR variants that functionally depend on the presence of doxycycline, mediated by a TetR-responsive aptamer ${ }^{17}$, which has been previously used in combination with the theophylline aptamer to control transcription ${ }^{18}$. Besides these strategies, modalities to sequester miRs ${ }^{19-21}$ or to inhibit their function by small molecules ${ }^{22}$ were developed and applied in cell culture and in vivo. Most of these approaches rely on the exogenous addition of small molecules, which per se might interfere with other biological processes, have limited availability and stability in vivo, suffer from diffusional spread, and are of restricted reversibility ${ }^{23}$. To overcome certain of these limitations, light-dependent control of regulatory RNA has also been described $^{24-26}$, but the pertinent approaches invariably require chemical synthesis and the exogenous addition of the modified RNAs to biological systems. By contrast, entirely genetic approaches to gain spatiotemporal control over regulatory RNA function remain elusive but are highly desirable, as they would offer a plethora of applications to precisely and reversibly control gene expression and downstream processes.

Here, we devise a fully genetically encodable, generic approach that achieves light-dependent control of pre-miR and shRNA activity. We construct chimeric RNA molecules consisting of mature miR and siRNA sequences conjoined with an RNA aptamer that binds to the light-oxygen-voltage (LOV) photoreceptor PAL in a light-dependent manner ${ }^{27,28}$. The chimeric RNAs enable the spatiotemporal control of short regulatory RNA function in mammalian cells, as we showcase for the lightdependent control of gene expression and cell-cycle progression. This hitherto unavailable modality establishes a versatile RNA control system for analyzing various protein and miR functionalities in a reversible, spatiotemporally resolved, and non-invasive manner, and with full genetic encoding. Owing to the modularity of the chimeric RNAs, the technology readily applies to neararbitrary shRNAs.

\section{Results}

PAL-mediated regulation of pre-miR activity. Our design of light-responsive pre-miRs anticipates an altered processivity of short regulatory RNAs by Dicer owing to light-activated binding of the LOV receptor PAL to the apical loop domain ${ }^{28-30}$. To implement this design, we embedded the cognate aptamer domain of PAL in the apical loop of short regulatory RNAs. We hypothesized that thereby regulatory RNA function can be controlled by blue light (Fig. 1).

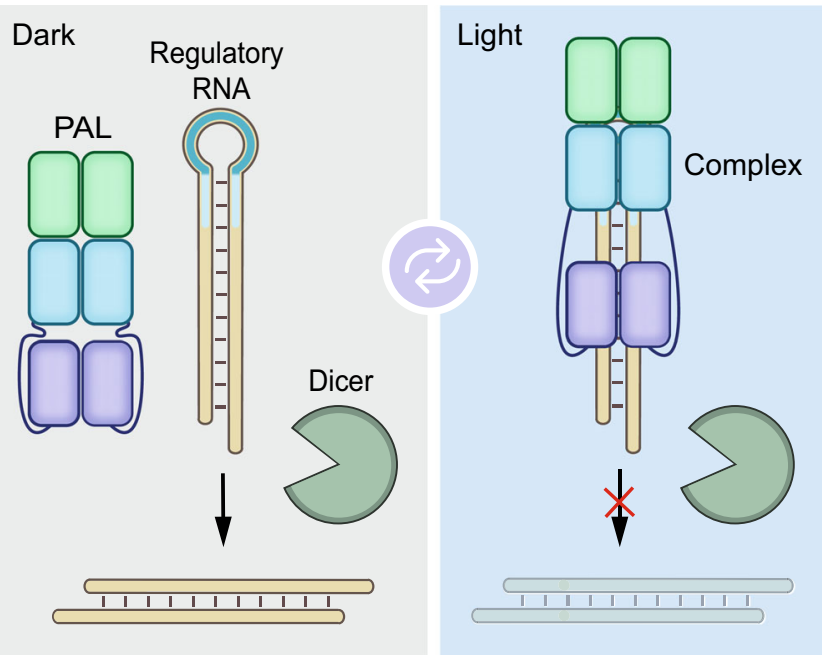

Fig. 1 General design of light-dependent regulatory RNAs. The PAL protein reversibly binds to its cognate RNA aptamer (highlighted in blue) embedded in the apical loop domain of a regulatory RNA (highlighted in light orange) in the light and thereby influences regulatory RNA function.

We generated pre-miR variants by replacing the apical loop domain with the PAL-binding RNA aptamer 53 (Fig. 2a, Supplementary Table 1). Notably, the RNA aptamer 53 interacts preferentially with the light-adapted state of PAL and to a much lesser extent with its dark-adapted conformation (Supplementary Fig. 3a, b). We first generated aptamer-modified variants of premiR-21 (SHA, Fig. 2a) and analyzed them in reporter gene assays that employ the expression of secreted Metrida luciferase or of enhanced green fluorescent protein (eGFP) with miR-21 target sites embedded in the $3^{\prime}$-UTRs of the respective mRNA (Supplementary Fig. 1a, b, 2) ${ }^{31}$. As controls, we constructed pre-miR-21 variants that bear a single point mutant (G11C) within the PAL aptamer that renders them binding-incompetent (SHC, SHD), a non-functional miR-21 domain (SHB, SHD) ${ }^{32}$, or with both domains altered (SHD, Fig. 2a). Interaction experiments in vitro revealed light-dependent binding of SHA and SHB to PAL, similar to the parental aptamer (53), whereas pre-miR variants with mutated aptamer domains (SHC, SHD) did not bind (Supplementary Fig. 3a, b). For all experiments, a transgenic HEK293 cell line stably expressing mCherry-PAL (HEK293PAL) at an average concentration of $1 \mu \mathrm{M}$ was used (Supplementary Fig. 4). The pre-miR-21 variants were transcribed under the control of the U6 promoter from plasmids ${ }^{33}$ co-transfected with the luciferase reporter. Whereas SHA supressed luciferase expression in darkness, irradiation with blue light $(\lambda=465 \mathrm{~nm})$ induced reporter gene expression by 4.4 -fold to $27 \%$ of the maximal value (Fig. 2b, d). Replacing either the miR-21 domain with a non-targeting RNA (SHB) or the aptamer domain by a non-binding point mutant (SHC) resulted in a loss of lightregulation (Fig. 2b, d, Supplementary Fig. 5). Likewise, the premiR21 variant having both RNA domains altered (SHD) neither supressed gene expression nor showed any light dependency (Fig. 2b, c). Analogous results were obtained for the eGFP reporter gene (Fig. 2d, e, Supplementary Figs. 7, 8, for details on eGFP gating strategy see Supplementary Fig. 6a), in that SHA inhibited expression in darkness, whereas a 4.4-fold induction of eGFP was observed in light (Fig. 2d, e). SHB and SHD did not inhibit eGFP expression, whereas SHC did, and none of the three variants exhibited light dependency (Fig. 2d). Intrinsic levels of argonaute 2 (AGO2) have been shown to limit RNA silencing efficiency ${ }^{34}$. Therefore, we co-expressed AGO2 and observed a more pronounced inhibition of eGFP expression by SHA and 
a

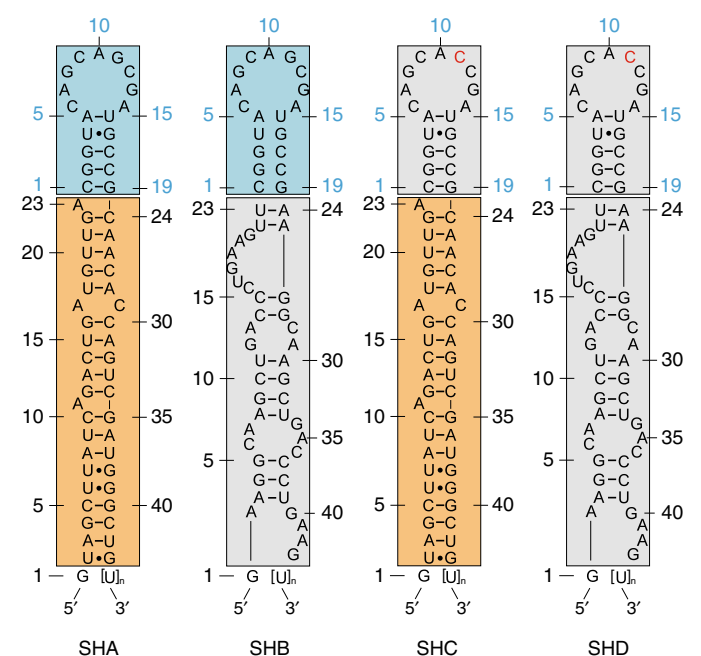

h

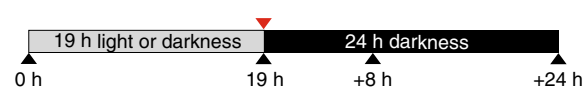

i

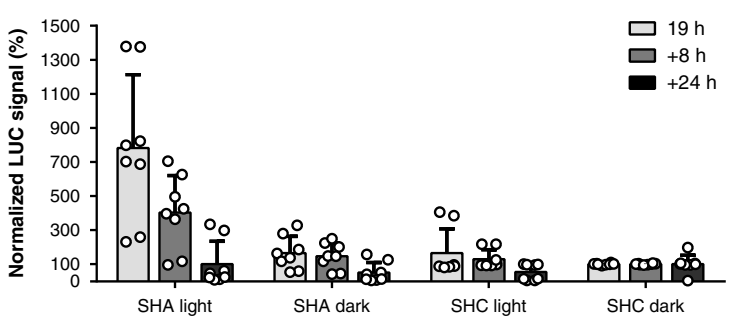

$\mathbf{k}$

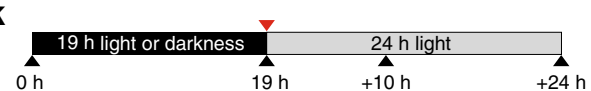

I

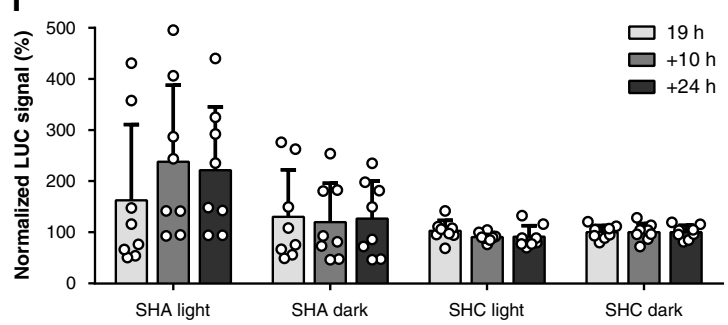

b
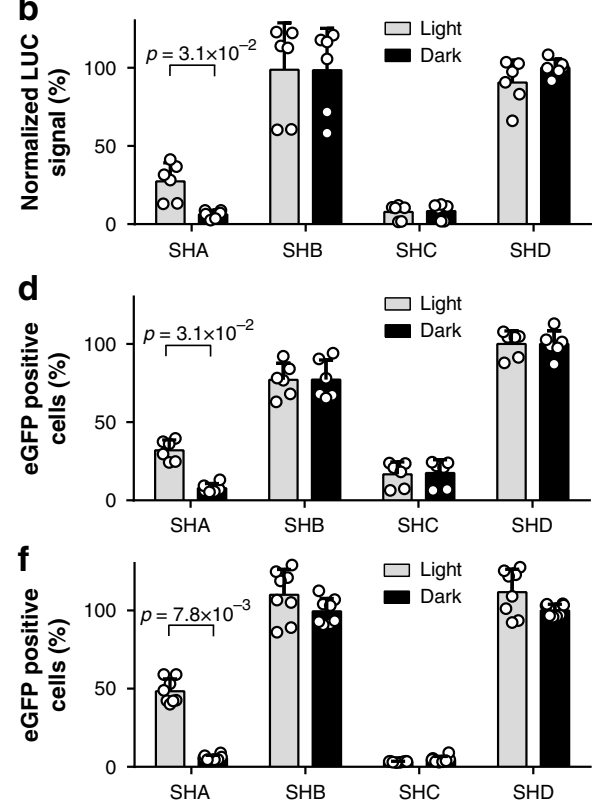

j

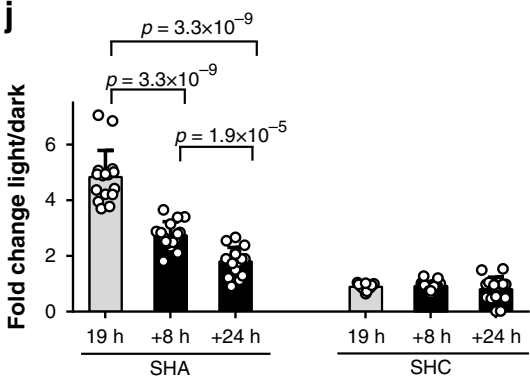

m

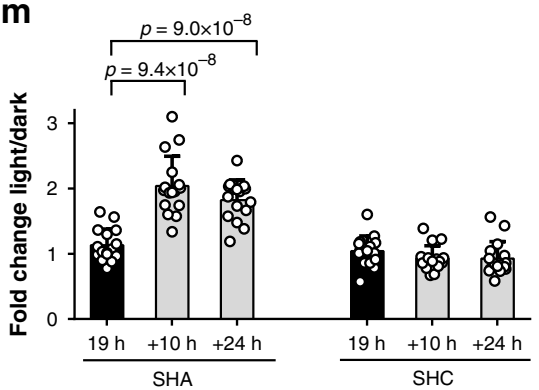

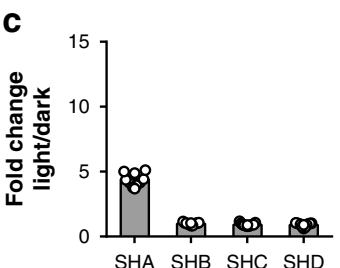

e
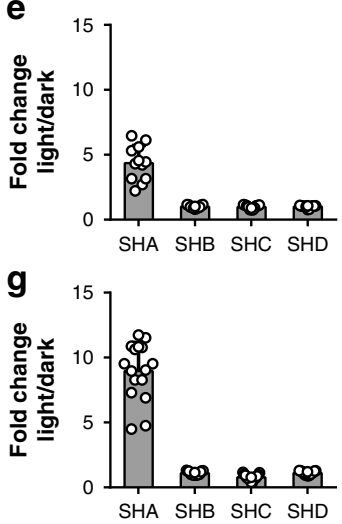

n
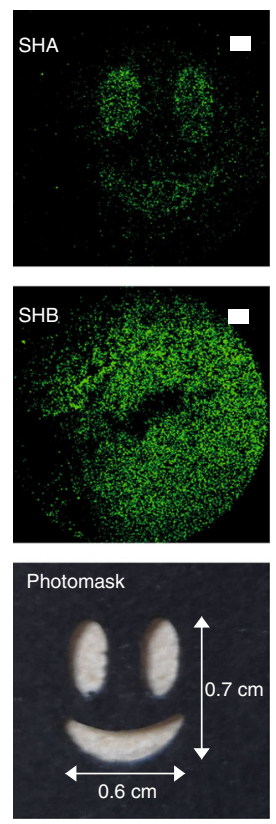

Fig. 2 A pre-miR21-aptamer chimera enables light-control of gene expression. a Schematic representation of the pre-miR21 variants and corresponding controls. Blue boxes: aptamer domain, orange boxes: miR21 domain, gray boxes: aptamer point mutant or control miR. b Luciferase expression after transfection of the indicated pre-miR21 variants. Values are normalized to SHD incubated in darkness. c Fold changes calculated from light vs. dark conditions from (b). d Number of cells expressing eGFP after transfection of the indicated pre-miR21 variants. Values are normalized to SHD incubated in darkness. e, Fold changes calculated from light vs. dark conditions from (d). f Number of cells expressing eGFP in the presence of elevated levels of AGO2 and after transfection of the indicated pre-miR21 variants. Values are normalized to SHD incubated in darkness. $\mathbf{g}$ Fold changes calculated from light vs. dark conditions from (f). $N=6(\mathbf{b}, \mathbf{d}), 8(\mathbf{f}), 12(\mathbf{c}, \mathbf{e})$ or $16(\mathbf{g})$. b-g Each biologically independent experiment was performed in duplicates. b, $\mathbf{d}, \mathbf{f}$ Gray bars: light conditions, black bars: dark conditions. c, e, $\mathbf{g}$ Dark gray bars: fold changes. b-f Wilcoxon two-sided signed-rank test was used for statistical analysis as a paired observation was assumed. $\mathbf{h}$ Illumination protocol applied in (i) and (j). i Luciferase expression level of cells expressing SHA or SHC. Shown are normalized values to SHC in darkness. $\mathbf{j}$ Fold changes calculated from light vs. dark conditions from (i). $\mathbf{k}$ Illumination protocol applied in (I) and (m). I Expression level of luciferase of cells expressing SHA or SHC. Shown are normalized values to SHC in darkness. $\mathbf{m}$ Fold changes calculated from light vs. dark conditions from (I). b-m Values are means \pm s. d. $N=8(\mathbf{i}, \mathbf{I})$ or $16(\mathbf{j}, \mathbf{m})$. Each biologically independent culture was performed in duplicates. $\mathbf{j}-\mathbf{m}$ Twosided Mann-Whitney $U$ test was used for statistical analysis as an unpaired observation was assumed. $\mathbf{n}$ Spatial patterning of eGFP expression after transfection with SHA (top panel) or SHB (middle panel). Irradiation was done on cells covered with a photomask (bottom panel); white bars: 1000 um. Source data for $(\mathbf{b}-\mathbf{g}, \mathbf{i}, \mathbf{j}, \mathbf{I}, \mathbf{m})$ are provided as a source data file. 
SHC in darkness (Fig. 2f, g, Supplementary Figs. 9, 10). Irradiation induced eGFP expression in the cells harboring SHA by 9 -fold (Fig. 2g). By contrast, experiments using SHB, SHC, and SHD did not reveal any light dependency (Fig. 2f, g).

We next assessed the reversibility of the approach using the luciferase reporter system. To this end, HEK293PAL cells harboring SHA were incubated for $19 \mathrm{~h}$ under blue light (Fig. 2h-j, Supplementary Fig. 11). Subsequently, the cells were kept in darkness for a further $24 \mathrm{~h}$. An increase of luciferase activity in the cell culture supernatants was observed after $19 \mathrm{~h}$ in light and a reduction when cells were kept in the dark afterwards (Fig. 2i, j). In turn, cells kept first in darkness did not reveal luciferase expression (Fig. 2k-m, Supplementary Fig. 12), but luciferase activity was detected when cells were subsequently exposed to light conditions (Fig. 2l, m). Cells having SHC did not reveal light-dependent luciferase expression (Fig. 2i, j, 1, m, Supplementary Figs. 11, 12). We also demonstrated spatial control of reporter gene expression using a photomask on HEK293PAL cells during irradiation (Fig. 2n). Expression of SHA resulted in eGFP expression predominantly in light-exposed areas, whereas eGFP expression was observed independently of the irradiation status in the presence of SHB (Fig. 2n).

To better characterize the processed miRs, we analyzed them by $3^{\prime}$ miR-RACE (rapid amplification of cDNA ends). Compared to reported natural pre-miR-21, we observed altered processing of SHA at the $3^{\prime}$ end of miR-21-5p (Supplementary Table 2). We attribute this observation to using the U6 promotor for pre-miR21 expression which requires an additional G-nucleotide for efficient transcription and, thus, induces altered Dicer processing 35 .

PAL-mediated regulation of shRNA activity. We next investigated whether the PAL-aptamer system can also be applied to shRNA molecules in a more generic manner to thereby enable versatile optogenetic control of RNA interference ${ }^{36}$. Initially, we constructed two shRNAs (SH1, SH2) that target different sites within the eGFP mRNA coding region (Supplementary Fig. 1c) and conjoined them with the PAL aptamer (Fig. 3a, b). The expression of eGFP in HEK293PAL cells harboring SH1 or SH2 was light-responsive, with $\mathrm{SH} 1$ being more efficient in eGFP suppression in the dark (Fig. 3c, d, Supplementary Figs. 13-16). As a control we used the miR-21-targeting SHA (Fig. 2a), which did not inhibit eGFP expression (Fig. 3c, d, Supplementary Figs. 13-17, for details on eGFP gating strategy see Supplementary Fig. 6b), as the miR target site is absent in the reporter mRNA employed in this experiment (Supplementary Fig. 1c). Structural variations of one or two nucleotides surrounding the Dicer cleavage site are common motifs found in natural pre-miRs and shRNAs ${ }^{37}$. These motifs alter the accuracy of shRNA processing and, thus, gene silencing efficacy ${ }^{38}$. We hence extended our study towards examining the impact of the nucleotides' identity in the hinge region that connects the siRNA with the aptamer domain on shRNA performance. To this end, we designed 8 variants with single nucleotide bulges in the hinge region of SH1 (Fig. 3d) located either up- (SH3, SH4, SH5, SH6) or downstream (SH7, SH8, SH9, SH10) of the aptamer domain (Fig. 3e). All variants demonstrated light-dependent induction of eGFP expression but with varying efficiency (Fig. 3f). An upstream C (SH6) or a downstream G (SH9) nucleotide, relative to the aptamer domain, revealed the lowest expression, upstream A (SH3) or G (SH5) nucleotides or downstream A (SH7), U (SH8) or C (SH10) nucleotides exhibited very similar properties. Likewise, the suppression efficiency of shRNAs in the dark varied among the constructs (Supplementary Figs. 13-15). The observed fold changes of eGFP expression (light $v s$. dark) are comparable across all shRNAs with SH9 having the lowest induction rate (Fig. 3g). In turn, an upstream $\mathrm{U}$ nucleotide (SH4) revealed similar fold changes (Fig. $3 \mathrm{~g}$ ) but a higher level of light-induced eGFP expression (Fig. 3f). Therefore, we chose $A$ and $U$ residues as representatives in the $\mathrm{SH} 2$ hinge region variants and included $\mathrm{G}$ (SH15) as a less efficient control. Single nucleotides inserted into the hinge regions of $\mathrm{SH} 2$ led to an improved eGFP knockdown in the dark (Fig. 3h, i, Supplementary Fig. 15), and all variants remained light-responsive. An adenine (SH11) or uridine (SH12) nucleotide at the hinge region upstream of the aptamer domain led to the highest number of eGFP-positive cells (Fig. 3i), and $\mathrm{SH} 14$ revealed the strongest increase of eGFP expression upon irradiation (15.3. fold) (Fig. 3j). SH16 with a mutated aptamer domain (G11C) did not reveal light-induced eGFP expression (Fig. 3h, i). Likewise, light-dependent induction of eGFP expression was also evident from fluorescence microscopy studies for the shRNA variants (Fig. 3k, Supplementary Fig. 17) $\mathrm{SH} 1$ (original hinge region), $\mathrm{SH} 3$ (intermediate performance), SH4 (highest number of eGFP positive cells when incubated in light), SH12 (second highest number of eGFP positive cells when incubated in light), and SH14 (highest light vs. dark fold change). In vitro binding studies verified light-dependent interaction with PAL of shRNA variants with engineered hinge regions (Supplementary Fig. 3a, b), indicating that these variations do not directly interfere with PAL binding but affect shRNA processivity. Of key importance, these findings testify to the modular design of the underlying chimeric RNAs and indicate that the domains for PAL-binding and mRNA-targeting are non-overlapping. As a corollary, we reasoned that near-arbitrary targeting domains should be accommodable with our technology.

Optoribogenetic control of cell-cycle progression. We hence extended our approach to regulating the expression of endogenous proteins via shRNAs. We chose cyclin B1 and CDK1 as targets, as they are both essential for the transition from the gap-2 $\left(\mathrm{G}_{2}\right)$ to the mitosis $(\mathrm{M})$ phase of the cell cycle ${ }^{39}$. Variations of the expression levels of cyclin B1 and CDK1 have phenotypic consequences and alter the distribution of cells in different stages of the cell cycle ${ }^{40,41}$. First, we generated shRNAs targeting cyclin B1 with varied hinge nucleotides, having either an adenine (SHCB1) or uridine (SHCB2) upstream or uridine (SHCB3) downstream of the aptamer domain (Fig. 4a). HEK293PAL cells having the shRNAs SHCB1-3 in darkness (i.e. cyclin B1 knockdown condition) accumulated in the $\mathrm{G}_{2} / \mathrm{M}$ phase (Fig. 4b, Supplementary Figs. 18, 19). Upon irradiation, the number of cells in $G_{2} / M$ phase was significantly reduced in cells having SHCB1 (Fig. $4 \mathrm{~b}$, Supplementary Figs. 18, 19), indicating the recovery of normal cell-cycle propagation. By contrast, propagation was not recovered upon irradiation for the binding-incompetent aptamer variants of SHCB1 (G11C, SHCB1m) (Fig. 4b). SHCB2 and SHCB3 did not affect cell-cycle propagation when irradiated (Fig. 4b). Based on these results, we constructed PALdependent shRNA variants of CDK1 having an adenine nucleotide in the hinge region upstream of the aptamer (SHCDK1, Fig. 4c). HEK293PAL cells having the shRNAs SHCDK1 in darkness also accumulated in the $G_{2} / M$ phase (Fig. 4d, Supplementary Figs. 18, 19). Upon irradiation, the number of cells in $\mathrm{G}_{2} / \mathrm{M}$ phase was significantly reduced (Fig. 4d, Supplementary Figs. 18, 19). Cells having the PALbinding deficient mutant shRNA SHCDK1m accumulated in the $G_{2} / M$ phase irrespective of the irradiation status (Fig. $4 \mathrm{~b}, \mathrm{~d}$, Supplementary Figs. 18, 19). No accumulation of cells in the $\mathrm{G}_{2} / \mathrm{M}$ phase was observed when cells expressed the nontargeting $\mathrm{SH} 3$ or were untreated (Supplementary Fig. 20). 
a

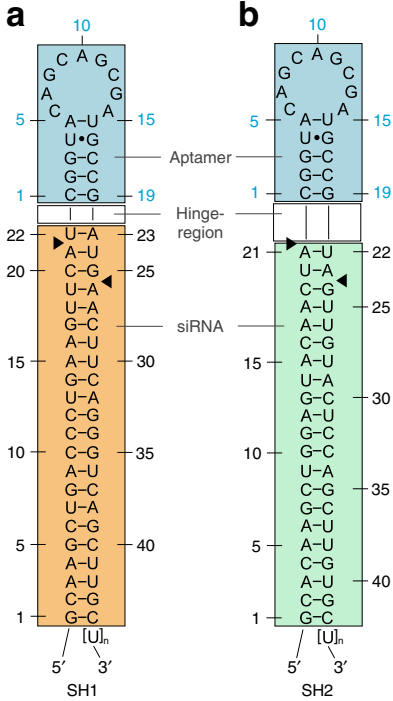

C

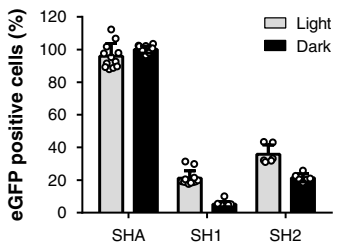

d

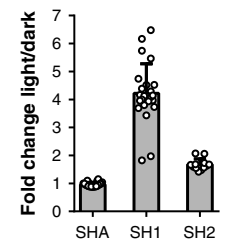

e

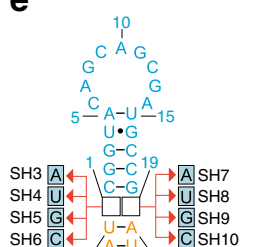

$22 \stackrel{C-G}{-13}$

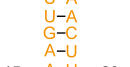

$15-A-\cup-30$

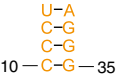

$10-C-G-35$

$G-C$
$U-A$

$5-\mathrm{G}-\mathrm{C}-40$

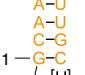

$5^{\prime} \stackrel{[U]_{n}}{3^{\prime}}$

h

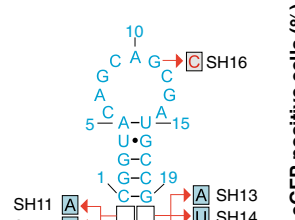

SH12

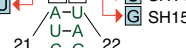

21 C-G 22

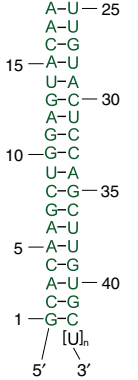

f

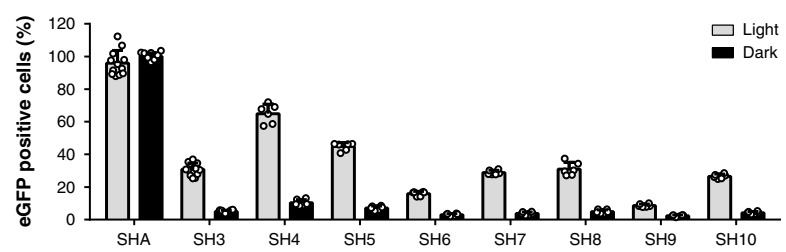

g

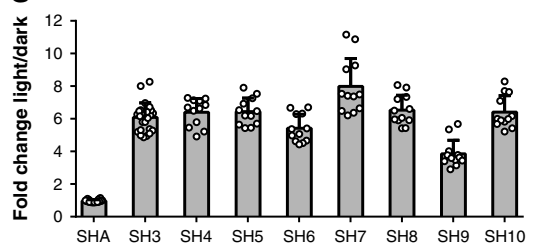

(1)
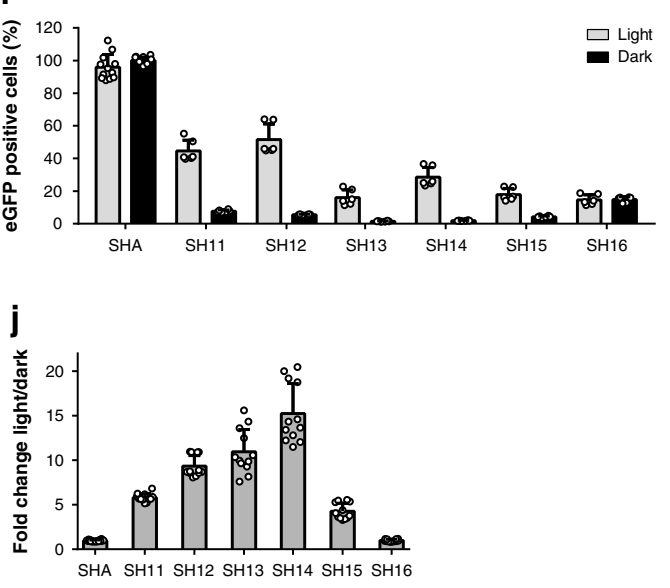

$\mathbf{k}$

$\mathrm{SH} 3$

$\mathrm{SH} 4$

SH14

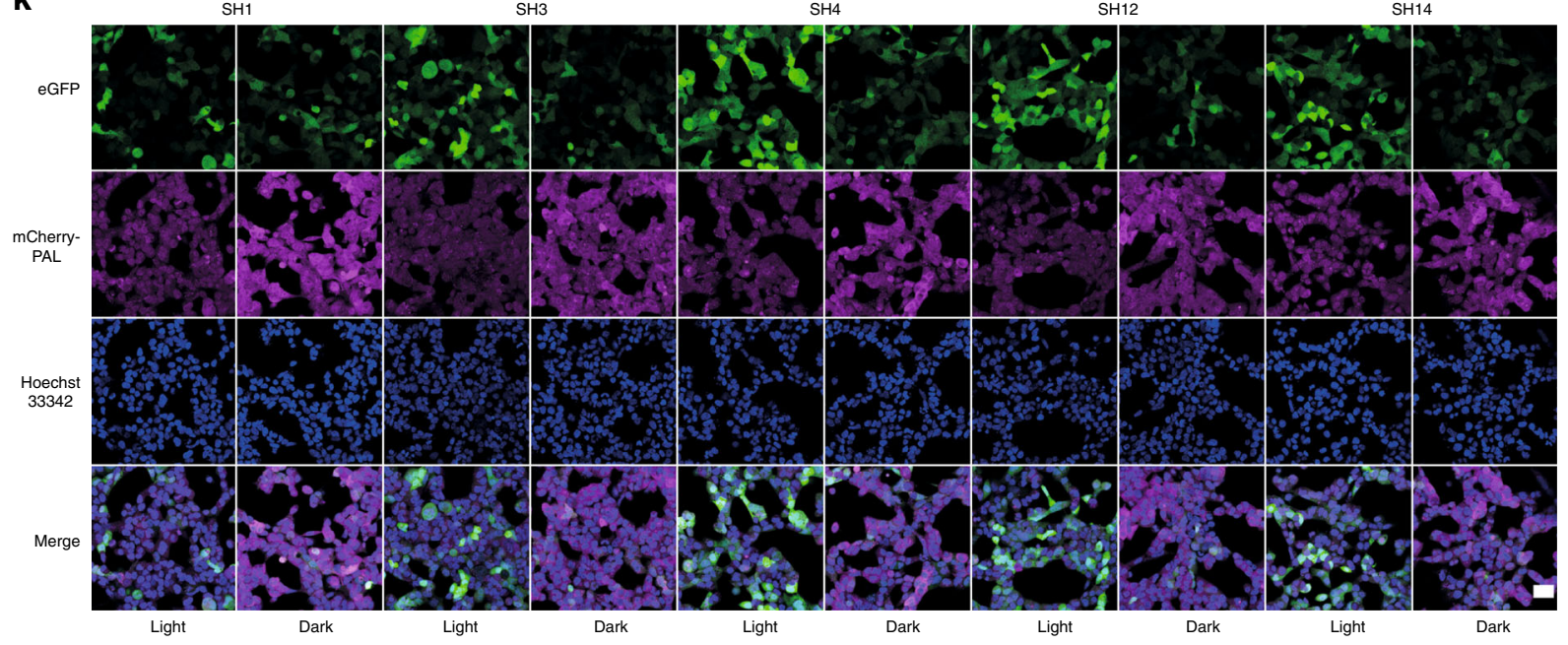

However, a slight accumulation of cells in $\mathrm{G}_{2} / \mathrm{M}$ phase was observed upon irradiation (Supplementary Fig. 20), most likely because of secondary irradiation effects on cells ${ }^{42}$. SHCB1 or SHCDK1 led to a decrease of cyclin B1 and CDK1 expression, respectively, which was reversed by irradiation (Fig. 4e-g, Supplementary Fig. 21). Variants of the shRNAs deficient for
PAL-binding (SHCB1m, SHCDK1m) suppressed protein expression independently of light (Fig. 4e-g). The nontargeting shRNA SH3 (Fig. 3b) did not affect cyclin B1 and CDK1 expression, the expression levels of both proteins were similar those when cells were untreated (Fig. 4e-g, Supplementary Fig. 21). Of note, the shRNA variants targeting cyclin 
Fig. 3 Design of shRNAs for the light-dependent expression of eGFP. Two different siRNA sequences SH1 (orange box, a) and SH2 (green box, b) targeting eGFP mRNA were conjoined with the PAL aptamer (blue boxes) as apical loop domains. Black arrows indicate a putative preferential dicer cleavage site ${ }^{44}$. c Number of cells expressing eGFP after transfection of SH1 or SH2. Values are normalized to SHA (Fig. 2a) in darkness. d Fold changes calculated from light vs. dark conditions from (c). e Single nucleotide permutations of the hinge region in SH1 and their impact on eGFP expression and light dependency (f). Values are normalized to SHA in darkness. g Fold changes calculated from light vs. dark conditions from (f). h Single nucleotide permutations of the hinge region in SH2 and their impact on eGFP expression and light dependency (i). Values are normalized to SHA in darkness. $\mathbf{j}$ Fold changes calculated from light vs. dark conditions from (i). k Fluorescence microscopy images of cells transfected with the indicated shRNA variants. Cells were incubated under either light or dark conditions. Scale bar: $40 \mu \mathrm{m}$. c, $\mathbf{f}, \mathbf{i} N=12$ (SHA, SH1, SH3) or 6 (all others). d, $\mathbf{g}$, j $N=24$ (SHA, SH1, SH3) or 12 (all others). c-k Each biologically independent experiment was performed in duplicates. Gray bars: light conditions, black bars: dark conditions. Dark gray bars: fold changes. Values are means \pm s.d. Source data for $(\mathbf{c}, \mathbf{d}, \mathbf{f}, \mathbf{g}, \mathbf{i}, \mathbf{j})$ are provided as a source data file.

a

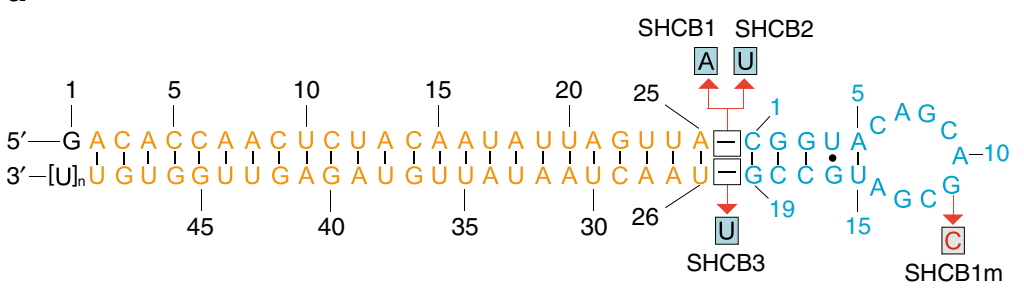

b

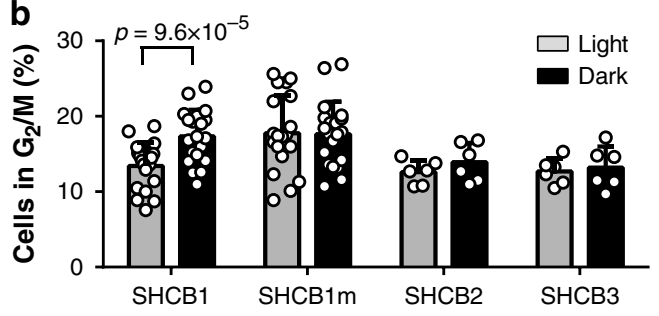

C

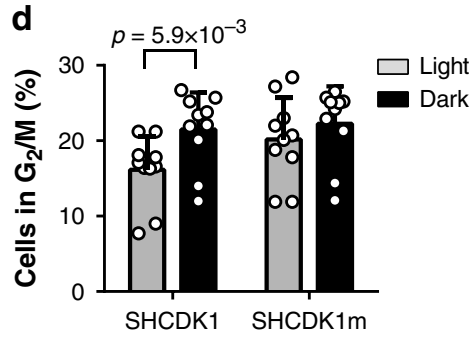

e

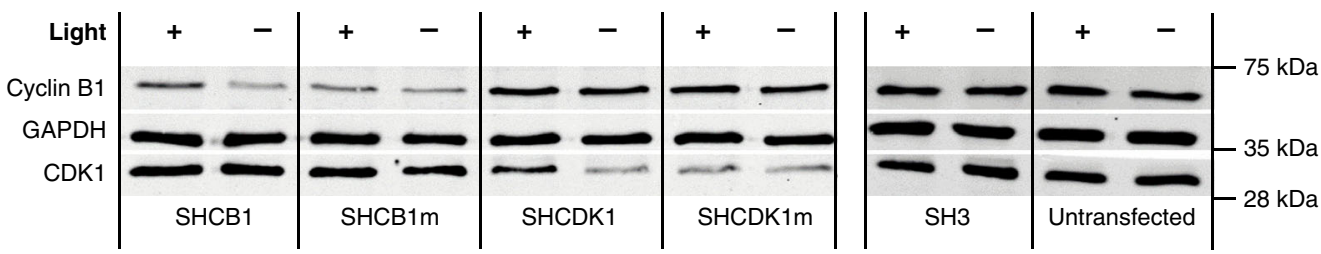

f

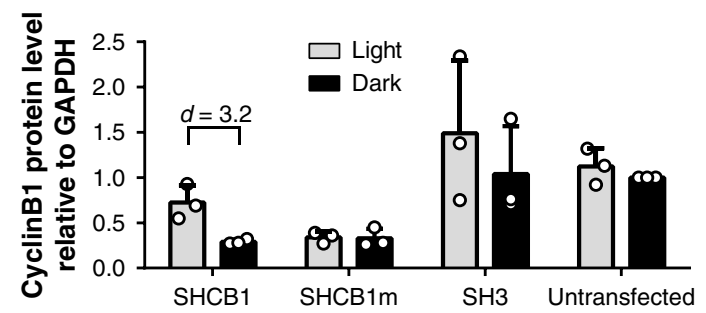

g

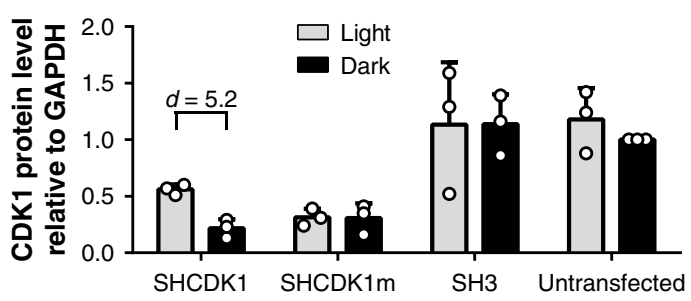

Fig. 4 Optoribogenetic control of the mammalian cell cycle. a shRNA variants used to control cyclin B1 gene expression. Blue: aptamer domain; orange: siRNA domain. b Percentages of HEK293PAL cells in $\mathrm{G}_{2} / \mathrm{M}$ phase of the cell cycle when transfected with indicated shRNAs targeting cyclin B1. c shRNA variants used to control CDK1 gene expression. Blue: aptamer domain; green: siRNA domain. $\mathbf{d}$ Percentages of HEK293PAL cells in $\mathrm{G}_{2} / \mathrm{M}_{\mathrm{B}}$ phase of the cell cycle when transfected with indicated shRNAs targeting CDK1. b $N=20$ (SHCB1, SHCB1m) or 6 (SHCB2, SHCB3). d N=10. b, d Each biologically independent experiment was performed in duplicates. $\mathbf{b}$ The identity of SHCB1 and SHCB1m was blinded and double-blinded in one experiment, each. $\mathbf{d}$ The identity of SHCDK1 and SHCDK1m was blinded and double-blinded in one experiment, each. b, d Wilcoxon two-sided signed-rank test was used for statistical analysis. e, Representative western blot image showing cyclin B1, CDK1 and GAPDH protein expression after transfection with the indicated shRNAs (for complete blots see Supplementary Fig. 21). e, $N=4$. e One biologically independent experiment was performed in duplicates, all others once. $\mathbf{f}, \mathbf{g}$ Quantification of cyclin B1 and CDK1 protein levels using pixel densitometry. $\mathbf{f}, \mathbf{g} N=3 . \mathbf{f}, \mathbf{g}$ All biologically independent experiments were performed once. $\mathbf{f}, \mathbf{g}$ Cohen's $d$ effect size was used for statistical analysis. Values were normalized to non-transfected cells incubated in darkness (Untransfected). b, d, f, $\mathbf{g}$ Gray bars: cells incubated under light conditions, black bars: cells incubated under dark conditions. Values are means \pm s.d. Source data for (b, d, $\mathbf{f}$, g) are provided as source data file. 
B1 did not affect CDK1 expression and vice versa (Fig. 4e, Supplementary Fig. 21).

\section{Discussion}

In conclusion, we demonstrate the fully genetically encodable light-control of miR and shRNA molecules in mammalian cells. The approach utilizes an aptamer that under blue light binds tightly and specifically to the photoreceptor protein PAL, and this interaction was shown to impact miR and shRNA function in regulating gene expression. We thus created an encoded onswitch, complementing a previously reported off-switch in which the PAL aptamer was embedded directly in the $5^{\prime} \mathrm{UTR}$ of mRNAs ${ }^{28}$. By offering full genetic encodability, reversibility, and noninvasiveness combined with a small genetic footprint (ca. 1.1 $\mathrm{kb}$ ), our approach transcends previous approaches for controlling regulatory RNA activity. Specifically, these features distinguish our method from ligand-gated techniques that invariably rely on the exogenous addition of specific compounds, thus abolishing full genetic encoding and limiting their application scope. Our method rivals CRISPR/Cas9-based approaches in its ready adaptability to target sequences through variation of the modular chimeric RNA. The technology thus unlocks optogenetic control of near-arbitrary gene products at the post-transcriptional level and expands the optogenetic toolbox. It should be noted that engineered pre-miR molecules might be more difficult to be approached, as genetic manipulation is required. Notably, the shRNA-based approach operates dominantly and can hence be used in wild-type cellular backgrounds, thus obviating the laborious construction of transgenic lines. To facilitate adoption of the technology, we investigated in detail sequence determinants affecting the efficiency of light regulation. We demonstrate that single nucleotide variations in the hinge region connecting the $\mathrm{miR} / \mathrm{siRNA}$ and the aptamer domains impact on regulatory RNA function and allow its fine-tuning, with an up to 15 -fold change in protein expression presently. Although we observed a preference for $\mathrm{A}$ and $\mathrm{U}$ nucleotides of the best-performing shRNAs, we recommend testing all canonical nucleotides (G,U,A, and C) at the hinge region, upstream and downstream of the aptamer domain to identify the most suitable variant. The induction of gene expression in light still reveals residual shRNA activity, depending on the regulatory RNA and target mRNA used. Further optimization might increase the dynamic range of the system, e.g., by varying the identity of the target region in a mRNA and, thus, the shRNA sequence. Besides light dependency, we also demonstrate spatial and temporal regulation and the suitability of the system to control endogenous proteins and cellular behavior, exemplified by controlling the cyclin $\mathrm{B} 1$ and $\mathrm{CDK} 1$ protein expression. This optoribogenetic approach extends to various shRNA and miR molecules for the investigation of dynamic biological processes by light, e.g., the relationship of proliferation and differentiation of neuronal stem cells, which depends on the progression of the cell cycle $e^{43}$. Additionally, optoribogenetic approaches may contribute to the understanding of dynamic micro RNA and protein functions that remain challenging to be resolved with the currently available methodologies.

\begin{abstract}
Methods
Molecular biology. All oligonucleotides were purchased from Ella Biotech, Planegg, Germany. Plasmid pIRESneo-FLAG/HA Ago2 was kindly provided by Thomas Tuschl. The plasmids pmCherry-C1, pMetLuc2-Control and peGFP-N1 were purchased from Takara Clontech. All miR and shRNAs described herein were cloned in the pSilencer 2.0-U6 plasmid backbone using restriction cloning. Corresponding $\mathrm{miR}$ and shRNA sequences are listed in Supplementary Table 1 . The
\end{abstract} identity of all constructs was confirmed by Sanger DNA sequencing (Eurofins).

PAL protein purification and RNA:PAL interaction assay. For protein expression, the plasmid pET-28c-PAL was transformed into ArcticExpress e. coli cells
(DE3, Agilent). Bacteria were grown in lysogeny broth (LB) medium supplemented with $50 \mu \mathrm{g} \mathrm{mL}^{-1}$ kanamycin, $20 \mu \mathrm{g} \mathrm{mL}^{-1}$ gentamycin and $50 \mu \mathrm{M}$ riboflavin at $37^{\circ}$ $\mathrm{C}$ at 120 r.p.m. until an optical density (OD) at $600 \mathrm{~nm}$ of 0.6 was reached, at which point expression was induced by addition of $1 \mathrm{mM}$ isopropyl $\beta-\mathrm{D}-1$ thiogalactopyranoside (IPTG). Incubation continued at $16^{\circ} \mathrm{C}$ and 120 r.p.m. for 66 $\mathrm{h}$, after which cells were harvested and lysed by ultrasound. The lysate was cleared by centrifugation and applied to an immobilized nickel ion affinity column (Marcherey Nagel). Protein was eluted in buffer A $(50 \mathrm{mM}$ Tris/ $\mathrm{HCl}, 200 \mathrm{mM}$ $\mathrm{NaCl}, 200 \mathrm{mM}$ imidazole, $10 \% \mathrm{w} / \mathrm{v}$ glycerol, $\mathrm{pH}$ 7.6). Protein was dialyzed into buffer B (12 mM HEPES/KOH, pH 7.2, $135 \mathrm{mM} \mathrm{KCl,} 10 \mathrm{mM} \mathrm{NaCl,} \mathrm{10 \%} \mathrm{w/v}$ glycerol). Identity of the protein was verified by polyacrylamide gel electrophoresis (PAGE). Protein concentration was determined by absorption spectroscopy using an extinction coefficient of $12,500 \mathrm{M}^{-1} \mathrm{~cm}^{-1}$ at $447 \mathrm{~nm}$.

In vitro transcription was performed using T7 RNA polymerase. DsDNA template coding for shRNA sequences was modified upstream by implementing T7 promoter sequence and two additional guanine residues (bold) after the transcription start site to ensure transcription (TAATACGACTCACTATAGGG, underlined G: transcription start site). DNaseI (Roche) digested RNA was pürified by PAGE and recovered by electroelution ( $8 \mathrm{M}$ ammonium acetate, $45 \mathrm{~min}, 150 \mathrm{~V}$ ). PAGE and electroelution were performed using TBE buffer (100 mM Tris, $100 \mathrm{mM}$ boric acid, $2 \mathrm{mM}$ ethylenediaminetetraacetic acid, $\mathrm{pH}$ 8.0).

For RNA:PAL interaction assays, PAL protein was biotinylated with a four-fold excess of EZ-Link Sulfo-NHS-LC-Biotin according to the manufacturer's instructions (Thermo Fisher Scientific) and coupled to streptavidin-coated wells of a plate (Pierce Streptavidin Coated Plates, Black, 96-Well, Thermo Fisher Scientific). Wells were washed three times with $200 \mu \mathrm{l}$ buffer B. Subsequently, 100 $\mu \mathrm{l}$ of $1.5 \mu \mathrm{M}$ biotinylated PAL in buffer B was added and the coupling was performed in darkness over night at $4{ }^{\circ} \mathrm{C}$. Afterwards, wells were washed three times with $200 \mu \mathrm{l}$ buffer B. In vitro transcribed pre-miR or shRNA constructs were incubated with immobilized PAL at the indicated concentrations in $100 \mu$ l buffer B for $30 \mathrm{~min}$ at $25^{\circ} \mathrm{C}$ under light $\left(\lambda_{\max }=465 \mathrm{~nm}, 2.15 \mathrm{~W} \mathrm{~cm}^{-2}\right)$ or in darkness, followed by three washing steps with $200 \mu \mathrm{l}$ buffer b for 3 min each. Fluorescence detection was performed by adding $150 \mu$ l RiboGreen reagent (Quant-iT RiboGreen RNA Reagent, Thermo Fisher Scientific), diluted 500-fold in $1 \times$ TE buffer $(10 \mathrm{mM}$ Tris/HCl, pH 7.5, $1 \mathrm{mM}$ ethylenediaminetetraacetic acid) to each well. After $1 \mathrm{~h}$ incubation in darkness, fluorescence intensity was measured on a Tecan Ultra plate reader (Tecan) at excitation and emission wavelengths of 500 and $525 \mathrm{~nm}$, respectively. Results were normalized to full length aptamer (53) incubated under light conditions.

LED array. Blue light was administered to cells in pulses (30 s light on, $30 \mathrm{~s}$ light off) by a custom LED array (illuminance at $106 \mu \mathrm{W} \mathrm{cm}{ }^{-2}$ ) with $\lambda_{\max }=465 \mathrm{~nm}$. The LED array was powered using a custom-built microcontroller. Cells were exposed to light immediately after transfection until they were subjected to further investigation.

Light plate apparatus (LPA). A custom-made replicate of the LPA was produced by Hanns-Martin-Schmidt and used for the photomask experiment.

Working with mammalian cell lines. HEK293 cells (CLS Cell Lines Service) were cultured in DMEM medium (high glucose, GlutaMAX), supplemented with $1 \%$ non-essential amino acids (NEAA), 1\% Sodium Pyruvate (Thermo Fisher Scientific) and $10 \%$ fetal calf serum (FCS, Sigma-Aldrich), at $37^{\circ} \mathrm{C}$ in a humidified $5 \%$ $\mathrm{CO}_{2}$ atmosphere and were passaged every 2-3 days. HEK293PAL cells were cultured 1 week before and after cell sorting with $1 \%$ penicillin/streptomycin and in presence of Geneticin (G418, $400 \mu \mathrm{g} \mathrm{mL}^{-1}$, Thermo Fisher Scientific). Mycoplasma testing was performed every three months using PCR detection (Minerva biolabs)

Transient transfection. Cells were transfected $24 \mathrm{~h}$ after incubation in darkness in $500 \mu$ DMEM supplemented with $1 \%$ NEAA and $1 \%$ Sodium Pyruvate. $1.5 \mu \mathrm{L}$ Lipofectamine2000 (Thermo Fisher Scientific) was used per well in a 24-well plate format. For transfection experiments involving AGO2, cells were transfected with $250 \mathrm{ng}$ plasmid DNA (e.g., pSilencer, AGO2 and pEGFP-N1 plasmid at a mass ratio of 2:2:1). For reporter assays without AGO2 overexpression, cells were transfected with $500 \mathrm{ng}$ plasmid DNA (e.g. pSilencer and pEGFP-N1 or PMetLuc2Control plasmid at a mass ratio of 100:1). For transfections of shRNAs targeting intrinsic mRNAs, $500 \mathrm{ng}$ of the corresponding pSilencer plasmid variant was transfected. Plasmids and Lipofectamine2000 were each diluted in $50 \mu \mathrm{l}$ Opti-MEM (Thermo Fisher Scientific) per well and incubated $5 \mathrm{~min}$ before mixing at room temperature. After $20 \mathrm{~min}$ further incubation at room temperature, $100 \mu \mathrm{l}$ transfection mix was added per well. Four hours after transfection, $60 \mu \mathrm{l}$ FCS were added per well.

Flow cytometry analysis. Flow cytometry was performed on a BD FACSCanto II (BD Biosciences). Data was processed using FlowJo version 9.6.3 software. Cells were initially gated with SSC and FSC channels for cell debris and single cell populations. For measuring eGFP, cells were additionally gated using the fluorescein isothiocyanate (FITC) channel to determine eGFP positive cells. For cell-cycle measurements, cells were additionally gated using Phycoerythrin (PE) channel to determine the 
percentage of cells in the different cell-cycle phases. At least 30.000 cells were analyzed from each sample. eGFP and propidium iodide were excited with a $488 \mathrm{~nm}$ laser and detected with a $530 / 30$ or $585 / 42$ filter set, respectively. mCherry was excited with a $633 \mathrm{~nm}$ laser and detected with a 660/20 filter set.

Isolation of monoclonal cells. For the generation of stable monoclonal HEK293 cell lines expressing mCherry-PAL (HEK293PAL), $10^{6}$ cells were seeded into each well of a 6 well plate and transfected by Lipofectamine2000 transfection on the following day using $2.5 \mu \mathrm{g}$ plasmid DNA and $8 \mu \mathrm{l}$ Lipofectamine2000. Four hours after transfection, the supernatant was discarded and cells were washed with PBS before the addition of $3 \mathrm{~mL}$ cell medium. After three days, the medium was supplied with $400 \mu \mathrm{g} \mathrm{mL}^{-1} \mathrm{G} 418$. One week before and after cell sorting cell medium was supplemented with $1 \%$ penicillin/streptomycin (Thermo Fisher Scientific). After five weeks of selection, single cells, which strongly express mCherry, were sorted via fluorescence activated cell sorting into a well of a 96-well plate. Cells were cultivated until $80 \%$ confluency was reached in a T-175 culture flask. Then, cells were frozen or used in further experiments. We did not notice any change in growth rate and cell fitness of the HEK293PAL cell line compared to HEK293 cells.

Luciferase reporter assays in mammalian cells. $1 \times 10^{5}$ HEK293PAL cells were seeded in two separate 24 -well plates per well. After $24 \mathrm{~h}$, cells were transfected according to the protocol described above. Transfected cells were incubated for 19 $h$ in the presence of blue light using the LED array or in darkness.

For reversibility assays, one control plate was kept constantly in darkness. Another plate was incubated under varying light conditions after transfection as indicated by the time lines shown in Fig. $2 \mathrm{~h}, \mathrm{k}$. Immediately before the light irradiation status was altered for one plate, cell medium was exchanged for both plates involved in the assay (plate incubated constantly in darkness and plate exposed to varying light conditions).

For the luciferase assay, $50 \mu \mathrm{l}$ of the cell culture supernatant was transferred to wells of a white 96-well plate (LUMITRAC 200, Greiner). Five microliters of luciferase substrate dissolved in buffer according to the manufacturer's instructions (Ready-ToGlow Secreted Luciferase, Takara Clontech) was added and the reaction was incubated for $3 \mathrm{~min}$ at room temperature. The luminescence signal was measured using an EnSpire plate reader (PerkinElmer) with an integration time of $5 \mathrm{~s}$.

For the static luciferase assay (Fig. 2b), values were normalized to control premiR transfection (SHD) incubated in darkness, where no influence on luciferase expression was expected. For the reversibility luciferase assay (Fig. 2i, l), values were normalized to aptamer point mutant pre-miR21 variant (SHC) incubated constantly in darkness, where no light dependency was expected. Normalization was performed to each time point.

Photomask experiment. $7.5 \times 10^{4} \mathrm{HEK} 293 \mathrm{PAL}$ cells were seeded in black 24 -well plates with clear bottom (VisionPlate, 4 titude). After $24 \mathrm{~h}$, cells were transfected using the protocol for AGO2 overexpression as described in the transient transfection section. Then, the plate was mounted onto the custom-made LPA device and irradiated with $10 \mu \mathrm{W} \mathrm{cm}{ }^{-2}$ of constant light $\left(\lambda_{\max }=465 \mathrm{~nm}\right)$. After $48 \mathrm{~h}$, cells were analyzed by confocal laser scanning microscopy (LSM 710) using a $10 \times / 0.45$ objective and image concatenation ( $10 \%$ overlay). Imaging was performed at $37^{\circ} \mathrm{C}$. eGFP fluorescence was visualized as green color and image histograms were adjusted to $5 / 10$ before.tiff picture export (Zen Black software, Zeiss). Image brightness was adjusted to +150 and image sizes were adjusted to $300 \times 300$ pixels using Adobe Photoshop CS5 software.

\section{eGFP reporter assays in mammalian cells. $1 \times 10^{5}$ HEK293PAL cells were} seeded in two separate 24 -well plates per well. After $24 \mathrm{~h}$, transfection was performed as indicated in the transient transfection section. For the miR21-based reporter assays, the $3^{\prime} \mathrm{UTR}$ of peGFP-N1 bearing miR21 binding sites (Supplementary Fig. 1b) was used. Cells were incubated for $44 \mathrm{~h}$ in the presence of blue light using the LED array or in darkness. Then, the cell supernatant was aspirated, cells were washed and resuspended in PBS $\left(25^{\circ} \mathrm{C}\right)$. The subsequent analysis was performed using flow cytometry. The percentage of eGFP positive cells was nor malized to control pre-miR transfection (SHD) incubated in darkness where no influence on eGFP expression was expected for miR experiments. For shRNA experiments, the percentage of eGFP positive cells was normalized to functional pre-miR21 transfection containing the PAL aptamer (SHA) and incubated in darkness as no influence on eGFP expression was expected due to the absence of miR21 binding sites in the $3^{\prime}$ UTR sequence of the eGFP mRNA.

Fluorescence microscopy of HEK293PAL cells. $5 \times 10^{4}$ cells were seeded in black 24 -well plates with clear bottom ( $\mu$-plate, ibidi). After $24 \mathrm{~h}$, cells were transfected using the protocol for AGO2 overexpression as described in the transient transfection section. Cells were incubated for $44 \mathrm{~h}$ in the presence of blue light $(465 \mathrm{~nm}$, $106 \mu \mathrm{W} \mathrm{cm} \mathrm{cm}^{-2}, 30 \mathrm{~s}$ pulses) or in darkness. Then, the supernatant was replaced by cell medium containing $5 \mu \mathrm{g} \mathrm{mL}^{-1}$ Hoechst 33342 . After $10 \mathrm{~min}$ incubation at $37^{\circ}$ $\mathrm{C}$, the supernatant was replaced with cell medium only. Next, cells were analyzed by confocal laser scanning microscopy (LSM 710, Zen Black software, Zeiss) using a $20 \times / 0.8$ objective. We generated pictures comprising of $1528 \times 1528$ pixels with a resolution of $0.19 \mu \mathrm{m}$ per pixel and a pixel dwell time of $2.11 \mu \mathrm{s}$ for imaging of each fluorophore. Fluorescence of mCherry (excitation (ex)/emission (em): 543/ 578-696 nm), Hoechst 33342 (ex/em: 405/410-494) and eGFP (ex/em: 488/ 494-574 nm) was monitored, respectively. Imaging was performed at $37^{\circ} \mathrm{C}$.

Western blot analysis. HEK293PAL cells were lysed $44 \mathrm{~h}$ after transfection in RIPA buffer (Thermo Fisher Scientific) containing $1 \mathrm{mM}$ Phenylmethylsulfonyl fluoride. Lysates were cleared by centrifugation $\left(14,000 \mathrm{~g}\right.$ for $\left.15 \mathrm{~min}, 4^{\circ} \mathrm{C}\right)$. Cleared lysates in Laemmli buffer were incubated at $95^{\circ} \mathrm{C}$ for $5 \mathrm{~min}$ before loading on SDSPAGE gels. Protein quantification was performed using the Pierce BCA protein assay kit according to the manufacturer's instruction (Thermo Fisher Scientific). $5 \mu \mathrm{g}$ of protein per lane was loaded onto 10 or $12.5 \%$ SDS-PAGE gels and blotted in Transfer Buffer $(2.5 \mathrm{mM}$ Tris, $2 \%(\mathrm{w} / \mathrm{v})$ glycine, $0.9 \mathrm{M}$ urea) onto a nitrocellulose membrane (GE Healthcare Life Sciences) using a Bio-Rad Trans-Blot SD Semi-Dry Transfer Cell (BioRad) for $75 \mathrm{~min}$ at $20 \mathrm{~V}$ and $30 \mathrm{~W}$. Membranes were blocked with TBS-T buffer (20 mM Tris/HCl, pH 7.6, $150 \mathrm{mM} \mathrm{NaCl,} \mathrm{0.05 \%} \mathrm{Tween} 20$ (v/v)) containing 5\% BSA (AppliChem, Western Blot grade) under agitation at room temperature for $1 \mathrm{~h}$. Blots were cut according to the protein ladder (Prestained Protein Ladder-Mid-range molecular weight $(10-180 \mathrm{kDa})$, abcam) in a way that all target proteins can be individually incubated with the respective primary antibody (mouse anti-cdc2 (CDK1), Cell signaling POH1, \#9116 (1:1000); mouse antiGAPDH, Santa Cruz Biotechnology sc-47724 (1:4000); goat anti-Cyclin B1, R\&D Systems AF6000 (1:1000)) at $4{ }^{\circ} \mathrm{C}$ overnight or at room temperature for $1 \mathrm{~h}$ in TBS-T containing 5\% BSA. Detection was performed with IRDye 800CW goat anti-mouse (Li-cor 926-32210), donkey anti-goat (Li-cor 926-32214) and goat anti-rabbit (Licor 926-32211) at a dilution of 1:15000, respectively.

Antibody-stained blot pieces were arranged, and fluorescence images were acquired using an Odyssey Imaging System's (Li-cor) $800 \mathrm{~nm}$ channel (ex/em: 785/ $810 \mathrm{~nm}$ ) to visualize bound $800 \mathrm{CW}$ secondary antibodies. Pixel densitometry of blot bands was performed using Fiji software (ImageJ) by creating rectangles of equal sizes for each sample lane followed by quantification of the area under the peak of each protein spot. Relative density was calculated by dividing values obtained from putative CDK1 or cyclin B1 protein bands through the values obtained from the putative GAPDH band of each lane. Relative density values were normalized to untransfected cells incubated in darkness.

Cell-cycle assay. $1 \times 10^{5} \mathrm{HEK} 293 \mathrm{PAL}$ cells per well were seeded in two separate 24well plates. $24 \mathrm{~h}$ after seeding, transfection was performed according to the transfection protocol for shRNAs targeting intrinsic mRNAs as indicated in the transient transfection section. Cells were incubated for $44 \mathrm{~h}$ in the presence of blue light $(465 \mathrm{~nm}$, $106 \mu \mathrm{W} \mathrm{cm} \mathrm{cm}^{-2}, 30 \mathrm{~s}$ pulses) or in darkness. $44 \mathrm{~h}$ after transfection, cells were fixed in $70 \%$ ice-cold Methanol in PBS and incubated for at least $30 \mathrm{~min}$ at $4{ }^{\circ} \mathrm{C}$ followed by RNAse A $\left(50 \mu \mathrm{g} \mathrm{mL}^{-1}\right)$ and propidium iodide (PI, $\left.50 \mu \mathrm{g} \mathrm{mL}^{-1}\right)$ treatment for $30 \mathrm{~min}$ at $37^{\circ} \mathrm{C}$ under mild agitation. Subsequent analysis of cell-cycle distribution quantified by PI fluorescence per cell was performed using flow cytometry.

mCherry quantification in HEK293PAL cells. $1 \times 10^{6}$ HEK293PAL cells were lysed in $250 \mu \mathrm{l}$ RIPA buffer (Thermo Fisher Scientific) containing $1 \mathrm{mM}$ Phenylmethylsulfonyl fluoride (PMSF). Lysates were cleared by centrifugation $(14,000 \mathrm{~g}$ for $15 \mathrm{~min}, 4^{\circ} \mathrm{C}$ ). Generation of a mCherry standard curve and fluorescence measurements were performed using mCherry Quantification Kit (BioCat) according to the manufacturer's instruction with the exception that RIPA buffer containing $1 \mathrm{mM}$ PMSF was used instead of mCherry Assay Buffer. For calculation of the concentration of mCherry-PAL a cellular volume of $4000 \mu^{3}$ was assumed and the cytoplasm covering $1 / 3$ of this volume.

Determination of $\mathbf{3}^{\prime}$ ends of artificial miR21-5p. $1 \times 10^{5}$ HEK293PAL cells were seeded in a 24 -well plate. $24 \mathrm{~h}$ later, transfection with SHA and luciferase reporter plasmid was performed and cells were further incubated in darkness. $19 \mathrm{~h}$ after transfection total RNA extraction with TRIzol was performed according to the manufacturer's instructions (Thermo Fisher Scientific). $1.5 \mu \mathrm{g}$ total DNase-treated RNA was poly-adenylated using Poly-A Polymerase according to the manufacturer's instructions (New England Biolabs). The reaction was purified by phenol/chloroform extraction followed by ethanol precipitation. Reverse transcription was performed using $1 \mu \mathrm{g}$ poly-adenylated cDNA, Bioscript Reverse Transcriptase according to the manufacturer's instructions (Bioline, Supplementary Table 3). After another round of phenol/chloroform extraction followed by ethanol precipitation, PCR amplification was performed using Taq Polymerase and specific primers (Supplementary Table 3). Cloning was performed using TOPO-TA cloning kit according to the manufacturer's instructions (Thermo Fisher Scientific). Plasmids were isolated from individual clones using Plasmid DNA purification kit (Marcherey Nagel) and sent for sequencing (Eurofins).

Calculation of fold changes. Light-dependent fold changes were calculated by dividing values (crude values for luciferase assay, percentage of eGFP positive cells for eGFP assays) of samples incubated under light conditions through samples incubated in darkness (duplicates) to obtain four values for each independent experiment. 
Blinded experiments. The identity of SHCB1, SHCB1m, SHCDK1, SHCDK1m and $\mathrm{SH} 3$ which are shown in Fig. $4 \mathrm{~b}, \mathrm{~d}$ and Supplementary Figs. 18 and 20 were blinded and double-blinded in one experiment, each. For the blinded experiment, the identity of these PSilencer plasmids have been blinded before transfection by a second person. For the double-blinded experiment, another experimenter performed the assay that was blinded by a third person in a similar way. Identity of the samples was confirmed after data evaluation between the experimenter and the person who performed the blinding.

Statistics and reproducibility. Prism 6.01 (GraphPad Software, Inc.) was used to generate graphs and calculate $P$ values. For all statistical analysis, no gaussian distributions were assumed due to limited sample sizes. Wilcoxon two-sided signedrank test was used to compare equally treated cell samples incubated under the indicated light conditions. Therefore, a paired observation was assumed. Two-sided Mann-Whitney $U$ test was used to compare light-dependent fold changes between differently treated groups (e.g. different time points). Therefore, an unpaired observation was assumed. Cohen's $d$ effect size was used to calculate effect sizes in Western Blot analysis derived from equally treated cell samples incubated under the indicated light conditions. In this case, sample size was too small to test for significance. Datasets are presented as mean \pm s.d., if not otherwise stated.

Reporting summary. Further information on research design is available in the Nature Research Reporting Summary linked to this article.

\section{Data availability}

Data are available in the main text, the Supplementary Information, or from the corresponding author upon request. Plasmids from Addgene (\#10822) were used in this study. Source data are provided with this paper.

Received: 29 July 2020; Accepted: 1 September 2020;

Published online: 24 September 2020

\section{References}

1. Meister, G. \& Tuschl, T. Mechanisms of gene silencing by double-stranded RNA. Nature 431, 343-349 (2004).

2. Zamore, P. D., Tuschl, T., Sharp, P. A. \& Bartel, D. P. RNAi: double-stranded RNA directs the ATP-dependent cleavage of mRNA at 21 to 23 nucleotide intervals. Cell 101, 25-33 (2000).

3. Hannon, G. J. RNA interference. Nature 418, 244-251 (2002).

4. Sambandan, S. et al. Activity-dependent spatially localized miRNA maturation in neuronal dendrites. Science 355, 634-637 (2017).

5. Wieland, M. \& Hartig, J. S. Improved aptazyme design and in vivo screening enable riboswitching in bacteria. Angew. Chem. Int. Ed. Engl. 47, 2604-2607 (2008).

6. Werstuck, G. \& Green, M. R. Controlling gene expression in living cells through small molecule-RNA interactions. Science 282, 296-298 (1998).

7. Boussebayle, A. et al. Next-level riboswitch development-implementation of Capture-SELEX facilitates identification of a new synthetic riboswitch. Nucleic Acids Res. 47, 4883-4895 (2019).

8. Topp, S. \& Gallivan, J. P. Emerging applications of riboswitches in chemical biology. ACS Chem. Biol. 5, 139-148 (2010).

9. Yen, L. et al. Exogenous control of mammalian gene expression through modulation of RNA self-cleavage. Nature 431, 471-476 (2004).

10. Mello, C. C. \& Conte, D. Revealing the world of RNA interference. Nature 431, 338-342 (2004).

11. Dorsett, Y. \& Tuschl, T. siRNAs: applications in functional genomics and potential as therapeutics. Nat. Rev. Drug Discov. 3, 318-329 (2004).

12. Kirkbride, R. C. et al. Maternal small RNAs mediate spatial-temporal regulation of gene expression, imprinting, and seed development in Arabidopsis. Proc. Natl Acad. Sci. U. S. A. 116, 2761-2766 (2019).

13. Bayer, T. S. \& Smolke, C. D. Programmable ligand-controlled riboregulators of eukaryotic gene expression. Nat. Biotechnol. 23, 337-343 (2005).

14. Szulc, J., Wiznerowicz, M., Sauvain, M.-O., Trono, D. \& Aebischer, P. A versatile tool for conditional gene expression and knockdown. Nat. Methods 3, 109-116 (2006).

15. Berger, S. M. et al. Quantitative analysis of conditional gene inactivation using rationally designed, tetracycline-controlled miRNAs. Nucleic Acids Res. 38, e168-e168 (2010).

16. Kumar, D., An, C.-I. \& Yokobayashi, Y. Conditional RNA interference mediated by allosteric ribozyme. J. Am. Chem. Soc. 131, 13906-13907 (2009).

17. Atanasov, J., Groher, F., Weigand, J. E. \& Suess, B. Design and implementation of a synthetic pre-miR switch for controlling miRNA biogenesis in mammals. Nucleic Acids Res. 45, e181-e181 (2017).
18. Ausländer, D., Wieland, M., Ausländer, S., Tigges, M. \& Fussenegger, M. Rational design of a small molecule-responsive intramer controlling transgene expression in mammalian cells. Nucleic Acids Res. 39, e155-e155 (2011).

19. Ebert, M. S., Neilson, J. R. \& Sharp, P. A. MicroRNA sponges: competitive inhibitors of small RNAs in mammalian cells. Nat. Methods 4, 721-726 (2007).

20. Fulga, T. A. et al. A transgenic resource for conditional competitive inhibition of conserved Drosophila microRNAs. Nat. Commun. 6, 7279-10 (2015).

21. Horwich, M. D. \& Zamore, P. D. Design and delivery of antisense oligonucleotides to block microRNA function in cultured Drosophila and human cells. Nat. Protoc. 3, 1537-1549 (2008).

22. Velagapudi, S. P., Gallo, S. M. \& Disney, M. D. Sequence-based design of bioactive small molecules that target precursor microRNAs. Nat. Chem. Biol. 10, 291-297 (2014).

23. Dickins, R. A. et al. Tissue-specific and reversible RNA interference in transgenic mice. Nat. Genet. 39, 914-921 (2007).

24. Mikat, V. \& Heckel, A. Light-dependent RNA interference with nucleobasecaged siRNAs. RNA 13, 2341-2347 (2007).

25. Shah, S., Jain, P. K., Kala, A., Karunakaran, D. \& Friedman, S. H. Lightactivated RNA interference using double-stranded siRNA precursors modified using a remarkable regiospecificity of diazo-based photolabile groups. Nucleic Acids Res. 37, 4508-4517 (2009).

26. Kala, A., Jain, P. K., Karunakaran, D., Shah, S. \& Friedman, S. H. The synthesis of tetra-modified RNA for the multidimensional control of gene expression via light-activated RNA interference. Nat. Protoc. 9, 11-20 (2014).

27. Christie, J. M. et al. Arabidopsis NPH1: a flavoprotein with the properties of a photoreceptor for phototropism. Science 282, 1698-1701 (1998).

28. Weber, A. M. et al. A blue light receptor that mediates RNA binding and translational regulation. Nat. Chem. Biol. 15, 1085-1092 (2019).

29. Lünse, C. E. et al. An aptamer targeting the apical-loop domain modulates primiRNA processing. Angew. Chem. Int. Ed. Engl. 49, 4674-4677 (2010).

30. Tsutsumi, A., Kawamata, T., Izumi, N., Seitz, H. \& Tomari, Y. Recognition of the pre-miRNA structure by Drosophila Dicer-1. Nat. Struct. Mol. Biol. 18, 1153-1158 (2011)

31. Pofahl, M., Wengel, J. \& Mayer, G. Multifunctional nucleic acids for tumor cell treatment. Nucleic Acid Ther. 24, 171-177 (2014).

32. Henique, C. et al. Genetic and pharmacological inhibition of microRNA-92a maintains podocyte cell cycle quiescence and limits crescentic glomerulonephritis. Nat. Commun. 8, 1829-15 (2017).

33. Carbon, P. et al. A common octamer motif binding protein is involved in the transcription of U6 snRNA by RNA polymerase III and U2 snRNA by RNA polymerase II. Cell 51, 71-79 (1987).

34. Börner, K. et al. Robust RNAi enhancement via human Argonaute-2 overexpression from plasmids, viral vectors and cell lines. Nucleic Acids Res. 41, e199-e199 (2013).

35. MacRae, I. J., Zhou, K. \& Doudna, J. A. Structural determinants of RNA recognition and cleavage by Dicer. Nat. Struct. Mol. Biol. 14, 934-940 (2007).

36. Fire, A. et al. Potent and specific genetic interference by double-stranded RNA in Caenorhabditis elegans. Nature 391, 806-811 (1998).

37. Warf, M. B., Johnson, W. E. \& Bass, B. L. Improved annotation of C. elegans microRNAs by deep sequencing reveals structures associated with processing by Drosha and Dicer. RNA 17, 563-577 (2011)

38. $\mathrm{Gu}, \mathrm{S}$. et al. The loop position of shRNAs and pre-miRNAs is critical for the accuracy of dicer processing in vivo. Cell 151, 900-911 (2012).

39. Jackman, M., Lindon, C., Nigg, E. A. \& Pines, J. Active cyclin B1-Cdk1 first appears on centrosomes in prophase. Nat. Cell Biol. 5, 143-148 (2003).

40. Johnson, N. et al. Compromised CDK1 activity sensitizes BRCA-proficient cancers to PARP inhibition. Nat. Med. 17, 875-882 (2011).

41. Groisman, I., Jung, M.-Y., Sarkissian, M., Cao, Q. \& Richter, J. D. Translational control of the embryonic cell cycle. Cell 109, 473-483 (2002).

42. Hockberger, P. E. et al. Activation of flavin-containing oxidases underlies light-induced production of $\mathrm{H}_{2} \mathrm{O}_{2}$ in mammalian cells. Proc. Natl Acad. Sci. U. S. A. 96, 6255-6260 (1999)

43. Zhao, C. et al. MicroRNA let-7b regulates neural stem cell proliferation and differentiation by targeting nuclear receptor TLX signaling. Proc. Natl Acad. Sci. USA. 107, 1876-1881 (2010).

44. Setten, R. L., Rossi, J. J. \& Han, S.-P. The current state and future directions of RNAi-based therapeutics. Nat. Rev. Drug Discov. 18, 421-446 (2019).

\section{Acknowledgements}

This work was supported by funds from the European Union ERC ('OptoRibo', 615381) to G.M., and the German Research Council (grants MA3442/5-1 and 5-2 to G.M., and MO2192/6-1 to A.M.). We thank Prof. Gruss for critical commenting on the cell-cycle data. The FACS core facility of the University Hospital Bonn is 
acknowledged for support in applying the gating strategy for the eGFP expression experiments.

\section{Author contributions}

S.P. designed the shRNAs, developed and performed all PAL-dependent experiments in mammalian cells and wrote the manuscript. C.M. performed the interaction studies of RNA molecules with PAL, M.C. performed the blinded studies, A.M. conceived the project and discussed experiments, G.M. conceived the study, supervised, discussed, designed the experiments, and wrote the manuscript.

\section{Funding}

Open Access funding enabled and organized by Projekt DEAL.

\section{Competing interests}

The authors declare no competing interests.

\section{Additional information}

Supplementary information is available for this paper at https://doi.org/10.1038/s41467020-18673-5.

Correspondence and requests for materials should be addressed to G.M.
Peer review information Nature Communications thanks the anonymous reviewer(s) for their contribution to the peer review of this work. Peer reviewer reports are available.

Reprints and permission information is available at http://www.nature.com/reprints

Publisher's note Springer Nature remains neutral with regard to jurisdictional claims in published maps and institutional affiliations.

(c) (i) Open Access This article is licensed under a Creative Commons Attribution 4.0 International License, which permits use, sharing, adaptation, distribution and reproduction in any medium or format, as long as you give appropriate credit to the original author(s) and the source, provide a link to the Creative Commons license, and indicate if changes were made. The images or other third party material in this article are included in the article's Creative Commons license, unless indicated otherwise in a credit line to the material. If material is not included in the article's Creative Commons license and your intended use is not permitted by statutory regulation or exceeds the permitted use, you will need to obtain permission directly from the copyright holder. To view a copy of this license, visit http://creativecommons.org/ licenses/by/4.0/.

(C) The Author(s) 2020 